\title{
La révolution continue : le Conseil constitutionnel est une juridiction... au sens de l'article 267 du traité sur le fonctionnement de l'Union européenne
}

(1) Majeure: selon l'article 267 du traité sur le fonctionnement de l'Union européenne (TFUE), « la Cour de justice de l'Union européenne est compétente pour statuer, à titre préjudiciel (...) sur l'interprétation des traités (...) et l'interprétation des actes pris par les institutions, organes ou organismes de l'Union. (...)

Lorsqu'une telle question est soulevée dans une affaire pendante devant une juridiction nationale dont les décisions ne sont pas susceptibles d'un recours juridictionnel de droit interne, cette juridiction est tenue de saisir la Cour ».

(2) Mineure : le Conseil constitutionnel est une juridiction nationale dont les décisions ne sont pas susceptibles d'un recours juridictionnel de droit interne.

(3) Conclusion : il doit donc renvoyer à la Cour de justice toute question d'interprétation du droit de l’Union européenne dont il a à connâttre dans l'exercice de sa compétence.

Résumée par ce syllogisme, la décision du Conseil constitutionnel du 4 avril 2013, M. Jeremy F.', aura du mal à être perçue comme révolutionnaire, même s'il est vrai que c'est la première fois que le juge constitutionnel français procède à un renvoi préjudiciel en interprétation à la Cour de justice. Le Conseil constitutionnel respecte le droit de l'Union européenne, sans pour autant explicitement affirmer qu’il était « tenu » de renvoyer à la Cour de justice².

Cette lecture volontairement simplificatrice ne saurait masquer les difficultés, plus ou moins visibles ou avouables, qu'un tel renvoi ne manquait pas de soulever. Du côté du visible, l'obligation imposée au Conseil constitutionnel par la loi organique, dans le cadre de la question prioritaire de constitutionnalité (QPC), de statuer dans un certain délai pouvait être considérée comme un obstacle dirimant au renvoi préjudiciel à la Cour de justice. Le délai pour que la Cour de justice statue, y compris selon une procédure d'urgence ou accélérée, empêche le Conseil constitutionnel de respecter le délai de 3 mois qui lui est imposé pour statuer sur une QPC. Dans l'affaire Jeremy F., le Conseil constitutionnel a été saisi le 27 février, il a rendu une décision de renvoi préjudiciel le 4 avril $^{3}$, la Cour de justice s'est prononcée le 30 mai $2013^{4}$ selon la procédure

C.C., n 2013-314P QPC, 4 avril 2013, M. Jérémy F. [Absence de recours en cas d'extension des effets du mandat d'arrêt européen question préjudicielle à la Cour de justice de l'Union européenne], JORF, 7 avril 2013, p. 5799.

2 Selon le Conseil constitutionnel, l'incertitude entourant l'interprétation d'un article de la décision-cadre sur le mandat d'arrêt européen « exige qu'il soit préalablement statué sur l'interprétation de l'acte en cause », il en déduit, qu'en application de l'article 267 du TFUE, « il y a lieu » de renvoyer une question préjudicielle à la Cour de justice (consid. 7).

3

C.C., n 2013-314P QPC, 4 avril 2013, précit.

$4 \quad$ C.J., 30 mai 2013, Jeremy F. c. Premier ministre, aff. C-168/13 PPU. 
d'urgence, la décision au fond du Conseil constitutionnel étant intervenue le 14 juin ${ }^{5}$. Le délai de trois mois pour statuer n'a donc pas été respecté.

Du côté de l'invisible, et selon une approche en termes de pouvoir, le renvoi préjudiciel d'une cour constitutionnelle à la Cour de justice présente une dimension politique dans la mesure où il pourrait traduire une certaine soumission de celle-là à celle-ci et donc une atteinte à la maitrise de l'interprétation des paramètres de constitutionnalité par le juge constitutionnel. Ce motif est d'ordre symbolique plutôt que rationnel. Le renvoi préjudiciel traduit sans doute plus un défaut de compétence pour juger de la question qui justifie le renvoi, qu'une soumission à l'autorité chargée de juger cette question, même si le renvoi implique que la décision prise par l'autorité compétente soit suivie par l'autorité de renvoi. Autrement dit, le renvoi préjudiciel peut être lu comme soulevant une question de compétence et non une question de hiérarchisation des juridictions. La relativisation de la logique de soumission impliquée par le renvoi préjudiciel provient encore de ce que seule la juridiction de renvoi est maîtresse de ce qui est renvoyé. La soumission, et donc le respect de ce qu'aura décidé l'autorité à laquelle l'on renvoie, n'intervient en définitive que s'il a été consenti à ce renvoi. L'autorité de renvoi demeure maittresse de ce à quoi elle devra se soumettre grâce à la liberté de choix dont elle dispose de renvoyer ou de ne pas renvoyer. Il sera d'autant plus facile de transmettre une question préjudicielle que le coût de cette transmission, en termes de perte de pouvoir, et donc d'atteinte à la liberté d'appréciation de la juridiction de renvoi, est faible. La reconnaissance d'une difficulté d'interprétation ou, selon une formulation consacrée, d'un acte clair offre une large marge d'appréciation à la juridiction de renvoi. La pertinence des motifs invisibles est donc relative. Le caractère favorable du bilan coût/avantage du renvoi préjudiciel explique sans doute que le Conseil constitutionnel, à l'instar d'autres cours constitutionnelles européennes ${ }^{6}$, aient décidé de renvoyer à la Cour de justice une question préjudicielle en interprétation.

L'insertion des cours constitutionnelles dans le lien procédural qui unit le juge national à celui de l'Union européenne témoigne sans conteste d'une tendance globale à un renforcement du respect du droit de l'Union. Les réticences d'ordre politiques tendent à se réduire face au respect du droit, fût-il de l'Union européenne. Ce qui semble d'ailleurs dominer aujourd'hui dans la jurisprudence du Conseil constitutionnel, ce sont moins les difficultés contentieuses liées à l'articulation des rapports entre le droit constitutionnel et le droit de l'Union qu'une banalisation de ce contentieux constitutionnel du droit de l'Union, comme en témoignent les deux décisions rendues dans l'affaire Jeremy B. Les risques de conflits se dissipent. Peut-être ne relèvent-ils plus que du

5 C.C., $n^{\circ}$ 2013-314 QPC, 14 juin 2013, M. Jérémy F. [Absence de recours en cas d'extension des effets du mandat d'arrêt européen], JORF, 16 juin 2013, p. 10024.

$6 \quad$ Tel est le cas en particulier de la Cour constitutionnelle italienne (ordonnances no 102 et 103 du 13 février 2008), de la Cour constitutionnelle belge alors «Cour d'arbitrage » ( $n^{\circ}$ 6/97, 19 février 1997, Rec., n 1, janvier-février 1-8/97, p. 77 et s.), de la Cour constitutionnelle autrichienne (B 2251, 2594/97 du 10 mars 1999), du Tribunal constitutionnel espagnol (ATC 86/2001, 9 juin 2011, dans une affaire concernant le mandat d'arrêt européen) et la Cour constitutionnelle de Lituanie (8 mai 2007 ayant donné lieu à un arrêt de la CJCE, aff. C-239/07). 
fantasme doctrinal ou de l'hypothèse d'école? Soyons toujours prudent face aux tentations de prédiction jurisprudentielle, quod principi placuit legis habet vigor.

Pour ce qui nous intéresse, l'affaire Jeremy F. s'est déroulée en trois temps. Dans un premier temps, le Conseil constitutionnel a adopté le 4 avril 2013 une décision de renvoi préjudiciel en interprétation à la Cour de justice, suite au renvoi par la chambre criminelle de la Cour de cassation d'une QPC portant sur l'article 695-46 alinéa 4 du code de procédure pénale. La numérotation de la décision QPC se voit ajouter la lettre « $\mathrm{P}$ », après le numéro de la décision, mais avant les lettres "QPC», afin de signaler sa spécificité7. Le renvoi à la Cour de justice s'accompagne en effet d'une décision de surseoir à statuer sur la QPC soulevée. Ce renvoi s'explique dans la mesure où la disposition législative contestée intervenait dans le domaine d'application du droit de l'Union. Plus précisément, l'article 695-46 alinéa 4 du code de procédure pénale constitue l'une des dispositions législatives introduites par le législateur dans ce code afin de tirer les conséquences dans l'ordre interne de la décision-cadre $n^{\circ}$ 2002/584/JAI du Conseil du 13 juin 2002 relative au mandat d'arrêt européen et aux procédures de remise entre Etat membre. Cette décision-cadre avait nécessité, après un avis du Conseil d'Etat du 26 septembre 2002 soutenant l'inconstitutionnalité de celle-ci ${ }^{8}$, une révision constitutionnelle, la révision constitutionnelle du 25 mars 2003, introduisant dans la Constitution un article 88-2 aux termes duquel « la loi fixe les règles relatives au mandat d'arrêt européen en application des actes pris par les institutions de l'Union européenne ». La révision permet ainsi l'application de la décisioncadre en France, tout en habilitant le législateur à en tirer les conséquences normatives dans l'ordre interne. C'est précisément dans le cadre de cette habilitation que le législateur a adopté la disposition contestée et, plus largement, les articles 695-11 à 695-51 du code de procédure pénale relatifs au mandat d'arrêt européen, issus de l'article 17 de la loi n 2004-204 du 9 mars 2004 portant adaptation de la justice aux évolutions de la criminalité.

L'article 695-46 du code de procédure pénale fixe les règles de procédure applicables aux décisions prises par les autorités judiciaires françaises, suite à la remise aux autorités d'un autre Etat membre de l'Union européenne d'une personne arrêtée en France en vertu d'un mandat d'arrêt européen émis par ces autorités. Son alinéa 4, qui est contesté devant le Conseil constitutionnel, prévoit que la chambre d'instruction, compétente dans cette situation, «statue sans recours (...) dans le délai de trente jours à compter de la réception de la demande». Les requérants dénonçaient comme contraire au principe d'égalité devant la justice et au droit à un recours juridictionnel effectif cette exclusion de tout recours contre la décision de la chambre de

C.C., nº 2013-314P QPC, 4 avril 2013, précit.

C.E., Ass. gén., 26 septembre 2002, avis sur la décision-cadre du Conseil de l’Union européenne du 13 juin 2002 relative au mandat d'arrêt européen et aux procédures de remise aux Etats membres, R.F.D.A., 2003, p. 465, E.D.C.E., n 54, p. 192.

Voir sur cet avis : H. LABAYLE, «Le contrôle de la constitutionnalité du droit dérivé de l’Union européenne. L'entraide judiciaire européenne au Palais Royal», R.F.D.A., 2003, pp. 442-469 ; A. ONDUA, «L'ordre constitutionnel français à l'épreuve de la décision-cadre du 13 juin 2002 sur le mandat d'arrêt européen », A.J.D.A., 2003, pp. 1368-1374. 
l'instruction. En l'espèce, l'appréciation de la régularité constitutionnelle de la disposition contestée soulève problème. Si la décision-cadre impose une décision dans le délai de 30 jours, ce qui est repris par le code de procédure pénale, elle ne dit rien sur la possibilité ou l'impossibilité d'un recours contre cette décision. L'on ne sait donc pas si cette absence de recours posée par le droit national est imposée par la décision-cadre ou si elle relève de la marge d'appréciation du législateur national. La résolution de cette question emporte des conséquences sur l'exercice du contrôle de constitutionnalité.

Si l'absence de recours est une conséquence nécessaire du droit de l'Union, le Conseil constitutionnel ne peut pas contrôler la constitutionnalité de la disposition contestée. Cette dernière est en effet couverte par l'article 88-2 de la Constitution, qui habilite le législateur à intervenir dans le domaine du mandat d'arrêt européen, tel qu'il est régi par le droit de l'Union, en dérogation éventuelle avec les autres dispositions constitutionnelles. Il est vrai, qu'en application de la jurisprudence consolidée Confiance dans l'économie numérique', il est possible d'opposer aux directives de l'Union européenne, et sans doute également à l'ensemble du droit dérivé de l'Union, par l'intermédiaire des lois nationales de transposition, d'exécution ou d'application, les règles ou les principes inhérents à l'identité constitutionnelle de la France. Cette possibilité n'est cependant ouverte qu'en l'absence d'une volonté autre du pouvoir de révision constitutionnelle ; un conflit avéré devant le juge constitutionnel entre le droit de l'Union et l'identité nationale pouvant de la sorte toujours être résolu par l'intervention du pouvoir de révision constitutionnelle. A propos des lois de transposition des directives et de l'obligation constitutionnelle de transposition, le Conseil constitutionnel considère en effet que «la transposition d'une directive ne saurait aller à l'encontre d'une règle ou d'un principe inhérent à l'identité constitutionnelle de la France, sauf à ce que le constituant y ait consenti ${ }^{10}$. Le pouvoir de révision constitutionnelle peut toujours habiliter de manière expresse le droit de l'Union, si ce n'est de porter atteinte, du moins à déroger à l'identité constitutionnelle de la France ou plus largement à la Constitution, ce qui découle, pour les engagements internationaux, du mécanisme de contrôle de constitutionnalité a priori institué par l'article 54 de la Constitution. La question du respect de cette identité n'est, en tout état de cause, pas en jeu dans la décision, à partir du moment où il existe une habilitation constitutionnelle expresse à déroger à la Constitution contenue dans l'article 88-2 de la Constitution. Aussi, si l'absence de recours est une conséquence nécessaire de la décision-cadre, la disposition législative qui l’institue entre-t-elle sous la couverture constitutionnelle spéciale de l'article 88-2 de la Constitution et ne saurait en conséquence être contrôlée sur le fond par le Conseil constitutionnel.

\footnotetext{
9 C.C., nº 2004-496 DC, 10 juin 2004, Loi pour la confiance dans l'économie numérique, Rec., p. 101, cons. 9, à lire avec C.C., $\mathrm{n}^{\circ}$ 2006-540 DC, 27 juillet 2006, Loi relative au droit d'auteur et aux droits voisins dans la société de l'information, Rec., p. 88, cons. 28. 
En revanche, si l'absence de recours relève de la marge d'appréciation des Etats, la solution est tout autre. Le droit de l'Union n'est alors pas affecté par le contrôle de constitutionnalité et le Conseil constitutionnel peut exercer un contrôle normal, c'est-à-dire comme s'il s'agissait d'une disposition législative dépourvue de tout lien avec le droit de l’Union, sur l'article 695-46 alinéa 4 du code de procédure pénale. La résolution de cette question relative au lien existant entre la décision-cadre et la disposition contestée est décisive. Si la seconde est nécessaire à l'application de la première, aucun contrôle n'est possible, si elle ne l'est pas, un contrôle de constitutionalité classique est possible. Il est donc nécessaire d'établir ce lien avant d'exercer le contrôle, comme ne manque pas de le préciser le Conseil constitutionnel ${ }^{11}$. Or, la résolution de cette question dépend de l'interprétation que l'on retient de la décision-cadre et donc du droit de l'Union. La décision-cadre se contente de prévoir que la décision en question « est prise au plus tard trente jours après réception de la demande ». Elle n'est pas explicite sur la possibilité de prévoir ou d'interdire un recours. La brièveté du délai pourrait cependant être interprétée comme limitant les possibilités de recours. Difficile d'ailleurs de ne pas savourer le croisement des différentes données de l'espèce alors qu'un délai imposé pour statuer de 30 jours pouvait être considéré comme excluant un recours... à plus forte raison qu'un même délai impératif de trois mois était supposé faire obstacle à un autre recours, le renvoi préjudiciel à la Cour de justice.

En présence de cette difficulté d'interprétation ${ }^{12}$, le Conseil constitutionnel précise que « conformément à l'article 267 du Traité sur le fonctionnement de l'Union européenne, la Cour de justice de l'Union européenne est seule compétente pour se prononcer à titre préjudiciel sur une telle question». Il en déduit «que, par suite, il y a lieu de la lui renvoyer et de surseoir à statuer sur la question prioritaire de constitutionnalité posée $»^{13}$. Il demande en substance à la Cour si les articles 27 et 28 de la décision-cadre s'opposent à l'existence d'un recours suspendant la décision de la chambre d'instruction. La Cour de justice doit en conséquence établir si la disposition législative contestée est ou n'est pas imposée sur ce point par le droit de l'Union. De la réponse de la Cour de justice dépend l'autorisation ou l'interdiction pour le Conseil constitutionnel, en vertu des habilitations constitutionnelles nationales, de contrôler une disposition législative d'application du droit de l'Union européenne. En s'autorisant une lecture quelque peu pernicieuse de cette question, l'on ne peut manquer de saisir une certaine ironie de la situation alors que le Conseil constitutionnel demande en substance si le droit de l'Union autorise qu'une décision judiciaire potentiellement privative de liberté puisse ne faire l'objet d'aucune possibilité de recours, ce qui constitue a priori une violation manifeste du droit au recours juridictionnel. Le juge constitutionnel français met ainsi en évidence une difficulté d'interprétation du droit de l'Union potentiellement problématique concernant le respect des droits fondamentaux.

\footnotetext{
11 Cons. 7.

12 Même si l'existence d'une difficulté d'interprétation n’est pas explicitement mentionnée dans la décision, elle en découle alors que le Conseil constitutionnel met en évidence dans la motivation de la décision l'incertitude découlant de l'énoncé de la décision-cadre.

Cons. 7.
} 
Le malaise ainsi généré n'est pas absent de l'arrêt du 30 mai 2013, Jeremy E. c. Premier ministre, rendu dans un second temps par la Cour de justice et se prononçant sur la question préjudicielle du Conseil constitutionnel ${ }^{14}$. Avant de s'attarder sur la réponse de la Cour, l'on se doit de remarquer que cette dernière ne soulève en aucun cas, dans le cadre de l'application de l'article $267 \mathrm{du}$ TFUE, la question de la nature juridictionnelle du Conseil constitutionnel et qu'elle admet la soumission du renvoi préjudiciel à la procédure d'urgence. Sur le fond, la Cour reconnait d'emblée que la décision-cadre ne «réglemente pas expressément» la possibilité de former un recours suspensif à l'encontre de la décision de l'autorité judiciaire et, en conséquence, que ce silence n'«empêche pas les Etats membres de prévoir un tel recours ou leur impose de l'instituer $»^{15}$. Une fois ce constat posé, la Cour apprécie si la décision-cadre contient toutes les garanties qui s'imposent dans le domaine concerné. Elle précise en effet, qu'au regard de l'article 13 de la Convention européenne des droits de l'homme, tel qu'interprété par la Cour de Strasbourg, et de l'article 47 de la Charte des droits fondamentaux de l'Union européenne, seule l'intervention de l'autorité judiciaire est exigée en matière d'extradition et non pas un double degré de juridiction. La Cour vérifie ainsi d'abord que le silence de la décision-cadre sur la possibilité d'un recours n'est pas contraire aux exigences posées par la Convention de 1950 et la Charte des droits fondamentaux de l'Union ${ }^{16}$. Ensuite, elle indique que les Etats peuvent prévoir un recours suspensif, ce qui relève de leur marge d'appréciation, afin de préserver leurs « règles constitutionnelle relatives, notamment, au respect du droit à un procès équitable ». Elle ajoute par ailleurs que l'utilisation par la décision-cadre des termes «décision définitive » n'exclut pas de recours contre cette décision. Les juges de Luxembourg explicitent enfin les limites de cette possibilité reconnue aux Etats membres de prévoir un recours suspensif. Il ne faut pas que la mise en place d'un tel recours remette en cause les délais impératifs fixés par la décision cadre de 30 à 60 jours selon les types de demandes adressées à l'autorité judiciaire et sur lesquelles cette dernière doit rendre sa décision. Pour ce qui intéresse le Conseil constitutionnel, le fait de prévoir ou de ne pas prévoir un recours suspensif relève de la marge d'appréciation des Etats membres, ce qui l'autorise à exercer un contrôle plein sur la disposition du code de procédure pénale contestée.

Dans un troisième temps, dans la décision du 14 juin 2013, le Conseil constitutionnel censure l'article 695-46 alinéa 4 du code de procédure pénale, après avoir pris soin de constater, conformément à l'arrêt de la Cour de justice, que cette disposition «ne découle pas

\footnotetext{
C.J., 30 mai 2013, Jérémy E. c. Premier ministre, aff. C-168/13 PPU.

$\int 38$.

Avant que l'arrêt de la Cour de justice ne soit rendu, Denys Simon envisageait la possibilité pour la Cour de transformer l'objet de la question préjudicielle dont elle était saisie, de l'interprétation vers l'appréciation de validité des dispositions de la décision-cadre, si ces dispositions étaient jugées irrégulières au regard des droits fondamentaux européens (V. D. Simon, « «Il y a toujours une première fois ». A propos de la décision 2013-314 QPC du Conseil constitutionnel du 4 avril $2013 »$, Europe, n 5, mai 2013, étude 5, S 30). Le fait qu'elles soient régulières explique sans doute qu'une telle transformation de l'objet du renvoi n'ait pas eu lieu. L'affirmation explicite de la régularité témoigne du doute qui pouvait exister, tout en l'écartant en substance.
} 
nécessairement des actes pris par les institutions de l'Union européenne relatifs au mandat d'arrêt européen ${ }^{17}$ et qu'elle n'est donc pas couverte par l'article 88-2 de la Constitution. La disposition contestée est considérée comme apportant «une restriction injustifiée au droit à exercer un recours juridictionnel effectif $»^{18}$. La déclaration d'inconstitutionnalité produit des effets immédiats dans tous les pourvois en cassation en cours à compter de la décision. L'origine européenne de la disposition contrôlée n'a donc emporté aucune incidence sur l'exercice du contrôle par le Conseil constitutionnel, précisément parce qu'elle n'était pas imposée par le droit de l'Union. La censure est donc neutre de ce point de vue. Elle l'est d'autant plus que la Cour de justice elle-même a retenu que la disposition législative contestée intervenait dans le domaine qui relève de la marge d'appréciation reconnue aux Etats par le droit de l'Union. La solution du Conseil constitutionnel est indiscutable du point de vue du droit de l'Union.

Au-delà de la complexité des données normatives de fond entourant le mandat d'arrêt européen $^{19}$, qui a donné lieu en l'occurrence à de nombreux contentieux dans d'autres Etats membres de l'Union ${ }^{20}$ et sur lesquelles nous ne sommes que brièvement revenus, l'affaire $M$. Jeremy constitue le premier renvoi préjudiciel adressé par le Conseil constitutionnel à la Cour de justice sur le fondement de l'article 267 du TFUE ${ }^{21}$. Elle tend à inscrire le Conseil constitutionnel dans le système juridictionnel prévu par le droit de l'Union faisant de la Cour de justice l'instance centralisatrice de l'interprétation et du respect du droit de l'Union grâce au lien procédural que constitue la question préjudicielle qui l'unit à tous les juges nationaux, juges de droit commun de l'application et du respect du droit de l'Union. Le Conseil constitutionnel n'est certes pas un juge du respect de ce droit, du moins en principe, conformément à la jurisprudence I.V.G. ${ }^{22}$, mais dans les situations dans lesquelles il a à en connaitre, il emprunte alors les voies de droit qui lui sont offertes par le droit de l'Union pour exercer cette mission. Ces situations dans lesquelles le Conseil constitutionnel peut connaître de l'application du droit de l'Union concernent les cas limités dans lesquels il se reconnaît compétent pour apprécier la conformité de la loi au droit de l'Union, c'est-à-dire pour le contrôle de constitutionnalité des lois de transposition des directives ${ }^{23}$ et celui de la loi organique sur le droit de vote et d'éligibilité des ressortissant de l'Union

17 C.C., n 2013-314 QPC, 14 juin 2013, précit., cons. 8.

18 Cons. 9.

19 Voir notamment pour plus de précision sur ce point : H. Labayle, R. Medhi, «Le Conseil constitutionnel, le mandat d'arrêt européen et le renvoi préjudiciel à la Cour de justice », RFDA, 2013, p. 461.

20 Voir en particulier le commentaire sous la décision du 4 avril en ligne sur le site du Conseil constitutionnel, pp. 9-11.

21 L'on remarquera en l'occurrence que les commentaires en ligne sous la décision du Conseil constitutionnel se concentrent sur la complexité du dispositif normatif du mandat d'arrêt européen, sans que la question du renvoi préjudiciel à la Cour de justice n'apparaisse, et ce à aucun moment, comme singulier, original ou même comme soulevant question. Le commentaire banalise ce qui apparaît sans doute comme la question dont la portée est la plus significative en droit du contentieux constitutionnel.

22 C.C., n ${ }^{\circ}$ 74-54 DC, 15 janvier 1975, I.V.G., Rec., p. 19, cons., réaffirmée en particulier avec force dans la décision du 12 mai 2010, Jeux de hasard, (n² 2010-605 DC) et pour la QPC dans la décision du 22 juillet 2010 (n² 2010-4/17 QPC).

23 C.C., n 2006-535 DC, 30 mars 2006, C.P.E., Rec., p. 50, cons. 28 ; n 2006-540 DC, 27 juillet 2006 , Loi relative au droit d'auteur et aux droits voisins dans la société de l'information, Rec., p. 88, cons. 16 et s. 
européenne aux élections municipales en application de l'article 88-3 de la Constitution ${ }^{24}$. Il faut également y ajouter tous ceux dans lesquels, comme dans l'affaire Jeremy P., le Conseil constitutionnel doit juger de la conformité à la Constitution d'une disposition nationale qui intervient dans le domaine ou pour l'application, la transposition ou l'exécution du droit de l'Union dans l'ordre interne. Dans ce dernier cas, l'interprétation du droit de l'Union ne renvoie pas à l'interprétation de la norme de référence du contrôle de constitutionnalité mais au lien existant entre le droit national, objet du contrôle, et le droit de l'Union, lien dont dépend le principe et/ou les modalités d'exercice du contrôle de constitutionalité. Dans toutes ces situations, l'acceptation potentielle du renvoi préjudiciel à la Cour de justice témoigne de ce que le juge constitutionnel se perçoit comme un juge d'application du droit de l'Union, même s'il n'est pas le juge de droit commun de ce droit.

Le constat de cette première expérience de renvoi préjudiciel à la Cour de justice pourrait se suffire à lui-même. La décision du Conseil constitutionnel comme son commentaire en ligne tendent d'ailleurs à banaliser ce renvoi. Celui-ci est certes justifié au fond par l'existence d'une difficulté d'interprétation mais, d'un point de vue procédural, le caractère inédit du renvoi préjudiciel ne transparait pas dans la décision, pas plus que sont évoquées, dans les commentaires en ligne, les décisions antérieures du juge constitutionnel refusant le principe du renvoi préjudiciel, du moins dans le cadre du contrôle a priori. Si la décision du 18 décembre 1997, Loi de financement de la sécurité sociale pour $1998^{25}$ pouvait faire l'objet de discussions, celle du 27 juillet 2006, Loi relative au droit d'auteur et aux droits voisins dans la société de l'information, est explicite, du moins dans le cadre du contrôle a priori: « devant statuer avant la promulgation de la loi dans le délai prévu par l'article 61 de la Constitution, le Conseil constitutionnel ne peut saisir la Cour de justice des Communautés européennes de la question préjudicielle prévue par l'article 234 du traité instituant la Communauté européenne $»^{26}$. Dans les commentaires en ligne, aucune difficulté qui aurait pu s'opposer au renvoi n'est évoquée; dans la décision, la question du délai pour statuer n'est mentionnée que pour justifier l'utilisation de la procédure d'urgence devant la Cour et non comme un obstacle au renvoi. Cette normalisation du renvoi préjudiciel ne doit pas pour autant occulter les interrogations que ce renvoi génère. Sous cet angle, il faut bien constater la neutralisation par le Conseil constitutionnel des obstacles politico-juridiques au renvoi préjudiciel à la Cour de justice $(\sqrt{ } \mathrm{I})$ pour pouvoir l'exercer de manière effective. De manière

24 C.C., $\mathrm{n}^{\circ}$ 92-312 DC, 2 septembre 1992, Maastricht II, Rec., p. 76, cons. 28 ; n 98-400 DC, 20 mai 1998, Droit de vote et d'éligibilité des ressortissants communautaires aux élections municipales, Rec., p. 251, cons. 4.

25 Dans la décision du 18 décembre 1997, Loi de financement de la sécurité sociale pour 1998 (nº 97-393 DC, Rec., p. 320), le Conseil constitutionnel a, semble-t-il, implicitement écarté sa compétence pour procéder à un renvoi à la Cour de justice. En jugeant que la signification de la directive évoquée par les requérants ne résultait pas «clairement » de cette directive, il s'est ainsi estimé incompétent pour interpréter des dispositions communautaires dont la signification n'était pas évidente. De plus, en présence d’une telle difficulté, il n’a pas procédé à un renvoi à la Cour de justice, se déclarant implicitement non visé par l'article 267 du TFUE (alors 234 du Traité C.E.) 
prospective, ce renvoi préjudiciel constitue par ailleurs un instrument de pacification potentielle des rapports de système ${ }^{27}(\mathbb{I I})$.

\section{§ I - La neutralisation des obstacles politico-normatifs au renvoi préjudiciel de l'article 267 TFUE par le Conseil constitutionnel}

Le renvoi préjudiciel à la Cour de justice se heurtait potentiellement à deux obstacles. Le premier, symbolique et d'ordre politique, consubstantiel au fait même de renvoyer une question préjudicielle en interprétation, résulte de la soumission consécutive du Conseil constitutionnel à la parole du juge de l'Union européenne. Le second, d'ordre juridique, conduit le Conseil constitutionnel à potentiellement statuer en méconnaissance des délais qui s'imposent à lui. Chacun de ces deux obstacles a été neutralisé par le Conseil constitutionnel. La neutralisation du premier est obtenue grâce à la justification du renvoi à partir de la compétence de la Cour de justice telle qu'elle est fixée par l'article 267 du TFUE (A). Celle du second est consécutive au choix du Conseil constitutionnel de ne pas retenir l'obligation de statuer dans un certain délai comme un obstacle décisif au renvoi préjudiciel (B).

\section{A - Un renvoi préjudiciel justifié par la compétence de la Cour de justice}

La soumission du Conseil constitutionnel à la parole du juge de Luxembourg est indiscutable dès lors que le premier procède à un renvoi préjudiciel en interprétation à destination de la seconde. La question préjudicielle est un instrument qui repose, dans sa mise en œuvre, sur la collaboration entre les juges nationaux et la Cour de justice de l'Union européenne. Elle ne participe toutefois pas d'un dialogue des juges ou d'une circulation des solutions juridiques ${ }^{28}$ car les juges de renvoi sont dans l'obligation de respecter la parole du juge auxquels ils ont renvoyé. Seule la pratique de ce renvoi permettra de mesurer le degré et les modalités de collaboration que le Conseil constitutionnel souhaite instaurer avec la Cour de justice. Le renvoi préjudiciel affecte en partie l'étendue du pouvoir du juge constitutionnel dans l'appréciation des éléments qui constituent son office. Il perd la maitrise, du moins en partie, de la norme objet ou de la norme de référence de son contrôle, selon les situations dans lesquelles le Conseil constitutionnel connait du droit de l’Union européenne.

\footnotetext{
27 Nous empruntons ici de manière volontaire l'expression de « rapports de système » plutôt que celle de « rapport entres les ordres juridiques» dans le sens substantiel défendu par Baptiste Bonnet dans son ouvrage sur la question (V. B. Bonnet, Repenser les rapports entre les ordres juridiques, Lextenso éditions, Forum, 2013, 208 p.), afin de marquer la nécessité de retenir une nouvelle approche nationale des rapports entre les ordres juridiques, s'appuyant sur la primauté du droit de l'Union sur le droit interne y compris constitutionnel. Cette nouvelle approche n'est nouvelle qu'en ce qu'elle s'éloigne de la lecture doctrinale nationale majoritaire souverainiste, mais elle n'est que la traduction d'une approche moniste internationaliste, qui n'a rien de nouvelle, des rapports entre les ordres juridiques. Sur ce dernier point, nous nous éloignons de la conception défendue par Baptiste Bonnet.

Voir sur cette notion: A. Le Quinio, Recherches sur la circulation des solutions juridiques : le recours au droit comparé par les juridictions constitutionnelles, Fondation Varenne, Collection des thèses nº 53, 2011, 522 p.
} 
La décision au fond du 14 juin 2013 témoigne de la volonté de respecter la parole de la Cour de justice et ce de manière rigoureuse. Le Conseil constitutionnel se considère lié par le seul dispositif de la décision du juge européen, c'est-à-dire par la réponse apportée à la question préjudicielle qui concrétise le résultat de l'office du juge tel qu'il est fixé par l'article $267 \mathrm{du}$ TFUE. Le Conseil constitutionnel reprend en l'occurrence le dispositif de l'arrêt de la Cour de justice, précédé de la mention selon laquelle la Cour «a dit pour droit que...» ${ }^{29}$. Le juge constitutionnel s'en tient ici à une lecture normativiste de l'autorité de chose jugée, réduisant la portée normative d'un jugement à ce qui a été jugé, à la seule norme, une norme individuelle et concrète posée par une décision de justice, et qui est contenue dans le dispositif de la décision. Ce n'est assurément pas celle qu'il retient de sa propre jurisprudence ${ }^{30}$. Cette soumission stricte et explicite à ce qu'a jugé la Cour de justice et, serait-on tenter d'ajouter, seulement à ce qu'elle a jugé, n'est cependant pas mise en avant par le juge constitutionnel. Au contraire, elle est banalisée si ce n'est omise, du moins dans la décision du 4 avril, et se pare de l'argument de la compétence de la Cour de justice pour interpréter le droit de l'Union.

La référence est brève, mais elle est explicite: «conformément à l'article 267 du Traité sur le fonctionnement de l'Union européenne, la Cour de justice de l'Union européenne est seule compétente pour se prononcer à titre préjudiciel $»^{31}$ sur une question d'interprétation d'un acte de l’Union. Cette référence à la compétence exclusive de la Cour de justice n'est pas inédite dans la jurisprudence du Conseil constitutionnel, en l'occurrence pour l'appréciation de la constitutionnalité des lois de transposition des directives ${ }^{32}$. Si le Conseil constitutionnel renvoie, ce n'est pas pour se soumettre à l'autorité de la Cour de justice, mais seulement pour respecter sa compétence. Il renvoie parce qu'il n'est pas compétent pour interpréter, non parce qu'il accepte de se soumettre à la parole d'un autre juge supposé supérieur. C'est d'ailleurs la nature même d'une question préjudicielle que de renvoyer à l'autorité compétente la résolution d'une question que l'on n'est pas habilité à résoudre. Il n'en reste pas moins vrai que le renvoi préjudiciel en interprétation, par son objet particulier, affecte la fonction première et ontologique du juge, à savoir sa capacité d'interpréter, ce qui n’est pas le cas d'un renvoi préjudiciel en appréciation de régularité.

Cette inscription du raisonnement du juge dans la perspective d'une répartition des compétences entre les juges masque, consciemment ou non, la soumission du Conseil constitutionnel à

29 C.C., $\mathrm{n}^{\circ}$ 2013-314 QPC, 14 juin 2013, précit., cons. 7.

30 Rappelons seulement ici, la jurisprudence classique du Conseil constitutionnel qui considère que l'autorité des décisions qu'il rend concerne non seulement le dispositif de celles-ci mais également les motifs qui en constituent le soutien nécessaire et le fondement même (C.C., nº 62-18 L, 16 janvier 1962, Nature juridique des dispositions de l'article 31 (alinéa 2) de la loi n 60-808 du 5 août 1960 d'orientation agricole Rec., p. 31, cons. 1). Voir par ailleurs sur la question de l'autorité des décisions du Conseil constitutionnel : «Sur un pont-aux-ânes ? L'autorité des décisions du Conseil constitutionnel, pour une distinction entre l'« autorité » et la « force » de chose jugée », RFDA, à paraitre, septembre 2013.

$31 \quad$ Nous soulignons.

32 Voir C.C., nº 2004-496 DC, précit., cons. 7. 
l'interprétation retenue par la Cour de justice. Il semble d'ailleurs possible, sans faire preuve d'une démarche trop constructive, d'étendre cette lecture en termes de compétence des rapports avec la Cour de justice aux rapports entre le droit interne et le droit de l'Union, tels qu'ils sont perçus par le Conseil constitutionnel. Sa jurisprudence témoigne d'un attachement à écarter toute appréhension en terme de hiérarchie des rapports entre le droit de l'Union et le droit interne dès lors qu'il s'agit d'appliquer le droit de l'Union, tout en maintenant ferme la hiérarchie, et plus exactement la primauté constitutionnelle, lorsqu'il s'agit d'affirmer le droit qui, dans l'ordre interne, dispose du dernier mot. Cette double dimension, répartition des compétences, lorsqu'il s'agit de reconnaître l'autorité du droit de l'Union, hiérarchie, pour affirmer la primauté du droit constitutionnel, n'est pas contradictoire. Il existe bien une répartition des compétences entre le droit de l'Union et le droit constitutionnel, mais c'est la Constitution qui la garantit. S'il est une norme, aux yeux du Conseil constitutionnel, qui fonde la répartition des compétences - telle que posée par les traités sur l'Union européenne - c'est la Constitution, qui se trouve «au sommet de l'ordre juridique interne », comme il l'a affirmé à plusieurs reprises ${ }^{33}$ et n'a pas manqué de la rappeler le commentaire sous la décision du 4 avril. La répartition des compétences entre la Constitution et le droit de l'Union est fixée par la Constitution elle-même. La Constitution (formelle) contient de la sorte les éléments de l'ordre répartiteur, qui garantit la répartition des compétences, et des éléments de l'un des deux ordres partiels qui font l'objet de la répartition, le droit interne formalisé dans la Constitution qui est soumis à cette répartition ${ }^{34}$.

Dans le prolongement, la soumission du juge de la Constitution à la parole d'un autre juge n'est possible que parce que, en dernière analyse, la Constitution le permet. Le raisonnement est éminent politique : point de droit en dehors de la Constitution. Il n'en est que plus symbolique et artificiel. Il est toujours possible de soutenir que c'est la Constitution qui décide de l'autorité et de la place du droit de l'Union dans l'ordre interne, nul ne concédera que c'est bien le droit de l'Union qui affecte profondément l'ordre interne, y compris constitutionnel. Le fait que le pouvoir de révision constitutionnelle ait consenti le principe de l'adhésion à l'Union, ce qui n'est pas discutable, n'y change rien : le droit constitutionnel cède au nom du respect du droit de l'Union au point que, désormais, le juge constitutionnel lui-même s'inscrit dans la procédure du renvoi préjudiciel de l'article 267 du TFUE. Certains opposeront encore l'existence de contrelimites, c'est-à-dire d'obstacles constitutionnels au respect du droit de l'Union européenne. Or, d'un point de vue juridique, ces contre-limites ont été désormais absorbées par le droit de l'Union lui-même, qui habilite les Etats en s'en prévaloir ce qui lui offre en contrepartie la capacité de les définir en vertu de l'article 4 \ 2 du Traité sur l'Union européenne (TUE). En prévoyant que le droit de l'Union respecte l'identité nationale, cette dernière notion est «européanisée » et son appréciation relève en conséquence de la compétence de la Cour de justice. En outre, en pratique, d'un point de vue quantitatif, les contre-limites paraissent bien dérisoires lorsqu'il s'agit de les

\footnotetext{
33 Voir en particulier implicitement C.C., n 2004-505 DC, 19 novembre 2004, Traité établissant une Constitution pour l'Europe, Rec., p. 173, cons. 13, et, explicitement, C.C., n 2007-560 DC, 20 décembre 2007, Traité modifiant le traité de l'Union européenne et le traité instituant la Communauté européenne, Rec., p. 459, cons. 8.

$34 \quad$ Voir sur cette lecture mais selon le point de vue de l’Union européenne : Théorie(s) du droit, Ellipses, 2008, \192.
} 
comparer à toutes les modifications profondes de l'ordre juridique interne, y compris constitutionnel, consécutives au droit de l'Union. Combien de limitations de souveraineté consécutives au droit de l'Union face à de symboliques contre-limites? La primauté constitutionnelle n'est plus qu'une primauté d'apparat. Dans un tel contexte, son affirmation constante apparait en réalité comme un aveu d'une soumission toujours plus importante et inexorable au droit de l'Union ${ }^{35}$.

\section{B - La neutralisation de l'obligation de statuer dans un certain délai}

La question du respect des délais pour statuer qui s'imposent au Conseil constitutionnel ne saurait a priori être considérée comme secondaire. Pour peu que l'on rappelle qu'il appartient à la Constitution, " au sommet de l'ordre juridique interne », éventuellement complétée sur son renvoi explicite par la loi organique, de fixer les compétences du juge constitutionnel et les modalités de leur exercice, l'on s'étonnera que celui-ci puisse s'affranchir des prescriptions de celle-là. Concernant la QPC, la loi organique prévoit un délai pour statuer de trois mois. La saisine de la Cour de justice, même si celle-ci statue en urgence, conduit le juge constitutionnel, comme c'était le cas en l'espèce, à méconnaître ce délai. A fortiori, si le Conseil constitutionnel transposait la solution retenue dans le cadre de la QPC au contrôle de constitutionnalité a priori, revenant ainsi sur sa jurisprudence antérieure ${ }^{36}$, le délai d'un mois qui s'impose ne serait pas non plus respecté. De plus, dans cette dernière situation, la promulgation d'une loi adoptée définitivement par le Parlement serait retardée de manière significative.

Le Conseil constitutionnel a choisi de manière implicite de contourner les textes plutôt que de les heurter de manière frontale en décidant de surseoir à statuer. De cette manière, le délai est suspendu. Le pouvoir que s'accorde le juge constitutionnel ne conduit pas à méconnaittre de manière frontale les délais qui lui sont imposés, mais à les suspendre pendant la durée de jugement de la question préjudicielle renvoyée à la Cour de justice. La situation n’est cependant pas prévue par les textes. Certains peuvent penser que cette capacité du juge de surseoir à statuer, pour être en mesure de juger le litige qui lui est soumis, est inhérente à sa fonction de juger. Le droit de l'Union et, plus précisément, la jurisprudence de la Cour de justice, sert sans doute également de fondement implicite à la reconnaissance de ce pouvoir par le juge constitutionnel. Les termes de l'arrêt Factortame méritent d'être rappelés ici : «le droit communautaire doit être interprété en ce sens que la juridiction nationale qui, saisie d'un litige, concernant le droit communautaire, estime que le seul obstacle qui s'oppose à ce qu'elle ordonne des mesures provisoires est une règle de droit national doit écarter l'application de cette règle $»^{37}$. La solution

\footnotetext{
35 Du moins tant que l'Union européenne existera et que les principes structurels d'intégration normative sur lesquels elle s'appuie seront maintenus, autrement dit, «à droit de l'Union européenne constant ». 
est transposable s'il faut reconnaître au Conseil constitutionnel le pouvoir de suspendre les délais de jugement qui s'imposent à lui afin de saisir la Cour de justice d'une question préjudicielle, cette dernière s'étant d'ailleurs prononcé en ce sens dans l'arrêt Melki et Abdeli3'. Il ne sera cependant pas soutenu ici que le Conseil constitutionnel est allé directement chercher dans le droit de l’Union jurisprudentiel une habilitation dont il ne disposait pas dans le droit national.

Cette reconnaissance par le Conseil constitutionnel de sa capacité de saisir la Cour de justice d'une question préjudicielle en interprétation malgré les délais qui s’imposent à lui pour juger dans le cadre de la QPC semble devoir être comprise comme une reconnaissance générale de cette capacité. En d'autres termes, il faut étendre cette solution au renvoi préjudiciel en appréciation de validité comme au contrôle de constitutionnalité a priori et à tout autre compétence du Conseil constitutionnel dans l'exercice de laquelle l'interprétation ou la régularité du droit de l'Union aurait à se poser. Rien ne justifie un fractionnement de cette capacité nouvelle en fonction de l'objet de la question préjudicielle ou du type de compétence du Conseil constitutionnel. Si l'on reprend la formule du juge constitutionnel, la référence à « l'article 267 du » TFUE est générale, sans distinction entre ses alinéas, tout comme la mention du fait que la «Cour de justice de l'Union est seule compétente pour se prononcer à titre préjudiciel» est générique. Cette formule est transposable, telle quelle, à une question préjudicielle en appréciation de validité. Il appartient au Conseil constitutionnel de décider et de revenir, le cas échéant, sur son affirmation explicite de l'impossibilité du renvoi dans le cadre du contrôle a priori ${ }^{39}$. En tout état de cause, le juge constitutionnel peut parfaitement se reconnaitre une compétence générale et ne jamais l'exercer ou ne le faire qu'avec parcimonie.

L'obligation de statuer dans un certain délai a cependant été retenue par le Conseil constitutionnel pour justifier la demande à la Cour de justice de mettre en œuvre la procédure d'urgence. Le juge constitutionnel a ajouté, à l'appui de ce motif, la privation de liberté dont le requérant a fait l'objet dans le cadre du procès principal ayant donné lieu à la QPC. La Cour de justice, qui apprécie si la procédure d'urgence doit ou ne doit pas être mise en œuvre, retient les deux arguments pour accepter la requête du Conseil constitutionnel. Il n'est donc pas certain que la seule question des délais puisse justifier le traitement en urgence de la question préjudicielle, ce qui apparaît problématique pour le contrôle de constitutionnalité a priori. La question parait d'autant plus décisive que le Conseil constitutionnel n'accepte de contourner les exigences de délai que parce que la Cour de justice statue en urgence. L'équilibre est ici fragile entre ce que le Conseil constitutionnel attend de la Cour de justice et ce que celle-ci est en mesure de lui accorder. Le principe de coopération loyale, consacré par l'article 4 \ 3 du TUE, ne manquera pas de garantir cet équilibre afin de permettre une saisine de la Cour de justice qui ne soit pas trop

38 C.J., 22 juin 2010, Aqiq Melki et Sélim Abdeli, C-188/10 et c-189/10, Rec. 2010 p. I-5667, \56 (« l'encadrement dans un délai strict de la durée d'examen par les juridictions nationales ne saurait faire échec au renvoi préjudiciel relatif à la validité de la directive en cause »). 
préjudiciable au respect des délais imposés pour le jugement de constitutionnalité. Il ne faut enfin pas oublier que la question est d'autant plus sensible que le domaine de la question préjudicielle d'urgence est limité par l'article 23bis du statut de la Cour de justice ${ }^{40}$ aux « renvois préjudiciels relatifs à l'espace de liberté, de sécurité et de justice ${ }^{41}$. Si le Conseil constitutionnel subordonne son renvoi à l'usage de la procédure d'urgence, les cas d'application seront limités à ce seul domaine même si celui-ci est large. C'est sans doute de ce côté là que la portée pratique de la décision du Conseil constitutionnel risque de demeurer limitée. Les conditions de mise en œuvre de la procédure accélérée, prévue par l'article 104bis du règlement de procédure devant la Cour de justice et qui peut concerner n'importe quel domaine du droit de l'Union, semblent a priori trop restrictives pour que cette autre voie puisse être empruntée par le juge constitutionnel. Une «urgence extraordinaire » doit en effet justifier le recours à la procédure accélérée. La Cour de justice l'avait cependant reconnue dans l'arrêt Melki et $A b d e l i^{42}$.

\section{$\S$ II - La pacification potentielle des rapports de systèmes}

L'usage du renvoi préjudiciel en interprétation par le juge constitutionnel favorise à l'évidence l'unité dans l'application du droit de l'Union. Lorsque le Conseil constitutionnel se trouve dans une situation contentieuse faisant intervenir le droit de l'Union européenne, le renvoi potentiel à la Cour de justice garantira une application uniforme de ce droit. Il peut également constituer de manière potentielle un instrument dynamique susceptible de pacifier les rapports de systèmes en marginalisant, voire en évitant, les conflits entre droit constitutionnel et droit de l'Union européenne. Cette conciliation possible peut résulter de l'utilisation tant du renvoi préjudiciel en interprétation (A) que de celui en appréciation de validité (B).

\section{A - L’usage conciliateur du renvoi préjudiciel en interprétation}

L'usage du renvoi préjudiciel en interprétation est en mesure de pacifier les rapports entre le droit national et le droit de l'Union dans deux situations contentieuses : dans la situation issue de la jurisprudence commentée Jeremy F. et dans le cadre de la jurisprudence C.P.E. ou en application de l'article 88-3 de la Constitution.

Dans cette dernière situation, le Conseil constitutionnel procède à un renvoi à la Cour de justice afin que celle-ci donne l'interprétation qu'il faut retenir du traité ou d'un acte de l'Union européenne qui sert de norme de référence au contrôle de constitutionnalité exercé par le juge

\footnotetext{
$40 \quad$ Ainsi que par l'article 104ter du règlement de procédure de la Cour de justice.

41 Voir, soulevant cette question : M. Gautier, «L'entrée timide du Conseil constitutionnel dans le système juridictionnel européen », AJDA, 2013, p. 1086.

42 Dans ces affaires, la Cour de justice était saisie par la Cour de cassation au titre de la procédure accélérée (art. $104 b i s$ du règlement de procédure de la Cour) et non de la procédure d'urgence (art. 104ter du règlement de procédure de la Cour).
} 
national. Toute l'habileté du Conseil constitutionnel consistera alors à poser une question à la Cour de justice mettant en évidence l'interprétation du droit de l'Union qui conduit soit à une censure de la loi, soit à une validation de celle-ci. Il mettra de la sorte la Cour de justice face aux conséquences éventuelles de sa réponse et ce sera en substance le juge européen qui sera à l'origine de la solution du juge national. A cet égard, même si la configuration contentieuse est différente, dans la décision du 4 avril, le Conseil constitutionnel a choisi de poser la question en retenant l'interprétation du droit de l'Union supposant une méconnaissance du droit constitutionnel national. Il demande à la Cour si le droit de l'Union s'oppose à un recours suspensif, ce qui marquerait à l'évidence une méconnaissance du droit au recours juridictionnel. La formulation négative, il est question d'interdiction et non d'autorisation par le droit de l'Union d'un recours suspensif, n'est pas neutre. Elle place la Cour de justice dans une situation défensive. Le juge européen doit valider ou invalider une situation conflictuelle et non pas une situation neutre. Il est encore possible d'envisager que l'interprétation donnée par la Cour de justice d'une disposition de l'Union européenne puisse conduire le Conseil constitutionnel à prononcer une réserve d'interprétation sur la disposition législative contrôlée pour en garantir la conformité à l’interprétation proposée par la Cour de justice.

Alors que les conditions d'application de la jurisprudence C.P.E. imposent au Conseil constitutionnel de vérifier au préalable que les dispositions de la directive susceptibles de servir de norme de référence dans le contrôle de la loi qui en assurent la transposition sont conformes aux règles et principes inhérents à l'identité constitutionnelle de la France, le Conseil constitutionnel pourrait également demander à la Cour de justice si les dispositions du droit de l'Union européenne en question doivent ou ne doivent pas être interprétées conformément à ces règles ou ces principes. A la Cour de justice de procéder à une interprétation conciliatrice entre les dispositions d'une directive, norme de référence du contrôle de constitutionnalité national, et l'identité nationale de la France permettant l'exercice du contrôle de conformité au droit de l'Union par le Conseil constitutionnel. Si les dispositions de la directive sont contraires à l'identité nationale, le juge constitutionnel ne pourra les utiliser comme norme de référence du contrôle. Au-delà de la solution contentieuse retenue par les juges, le constat d'une méconnaissance de l'identité n'est pas sans soulever des questions d'ordre politique plus larges. Une telle méconnaissance, on le verra, pourrait d'ailleurs également conduire le juge constitutionnel à soulever une question préjudicielle en appréciation de validité à la Cour de justice.

Dans le cadre de la jurisprudence Jeremy F., le renvoi préjudiciel est utilisé par le juge constitutionnel pour établir le lien existant entre la loi contrôlée et le droit de l'Union que celle-ci est censée appliquer. De ce lien, qu'il appartient à la Cour de justice d'expliciter par le renvoi préjudiciel dont elle est saisie, dépend le principe et/ou les modalités d'exercice du contrôle de constitutionnalité. Pour résumer, selon le lien existant, trois types de situation peuvent voir le jour: l'absence de contrôle de constitutionnalité, un contrôle de constitutionnalité limité au respect de l'identité nationale de la France ou un contrôle intégral. 
La première situation se rencontre lorsqu'une une disposition législative est nécessaire à l'application du droit de l'Union et que le domaine dans lequel intervient le droit de l'Union fait l'objet d'une habilitation constitutionnelle spéciale, comme c'est le cas dans l'affaire Jeremy F. La seconde situation intervient lorsqu'une disposition législative est nécessaire au droit de l'Union et qu'elle est contestée au regard des règles ou principes inhérents à l'identité constitutionnelle de la France $^{43}$. La situation est ici la plus conflictuelle alors que le droit constitutionnel est en mesure de faire obstacle à l'application du droit de l'Union. L'absence de contrôle au regard des autres principes constitutionnels, que l'on peut qualifier d'ordinaires, s'explique par l'existence de la couverture constitutionnelle générale dont dispose dans son ensemble le droit de l'Union européenne sur le fondement de l'article 88-1 de la Constitution. La dernière situation se rencontre lorsque la disposition législative contestée n'est pas nécessaire à l'application du droit de l'Union. Grâce au renvoi préjudiciel en interprétation, le Conseil constitutionnel ne règle pas lui-même la question de savoir le type de contrôle qu'il doit exercer, mais renvoie au juge suprême de l'Union européenne le soin de le faire.

Dans chacune de ces situations, la capacité de la Cour de justice à neutraliser le conflit est variable. Elle pourra désamorcer le conflit potentiel en jugeant que la difficulté constitutionnelle soulevée par le droit national, comme la Cour l'a fait dans l'affaire commentée, n'est pas consécutive à l'application du droit de l'Union et relève de la marge d'appréciation de l'Etat. La marge de manœuvre du juge européen n'en est pas moins réduite. Déterminer ce qu’impose le droit de l'Union ne laisse pas toujours une marge d'interprétation large. Celle-ci peut même inexistante, en particulier lorsque le droit national ne fait que reprendre les termes contenus dans un acte de l'Union, ce qui est loin d'être inédit en pratique. Il reste que, lorsque la mesure nationale n'est pas nécessaire au droit de l'Union, le contrôle du juge constitutionnel est indifférent. En présence d'une couverture constitutionnelle spéciale, la réponse de la Cour est en tout état de cause neutre, soit que le juge constitutionnel ne contrôlera pas la loi nécessaire au droit de l'Union car elle est couverte par une habilitation constitutionnelle spéciale, soit que le juge constitutionnel exercera un contrôle sur une disposition législative qui relève de la marge d'appréciation des Etats. C'est donc en cas d'atteinte éventuelle à l'identité nationale que l'interprétation de la Cour de justice sera la plus décisive. Le renvoi par le Conseil constitutionnel le sera tout autant, comme la formulation de la question par celui-ci. Il peut ainsi demander à la Cour de justice si le droit de l'Union mis en cause par l'intermédiaire du droit national doit être interprété dans un sens qui sera potentiellement celui susceptible de se heurter à l'identité nationale ou, au contraire, dans un sens qui permet de préserver l'identité nationale. Il appartiendra alors à la Cour de justice de décider en dernier lieu de l'atteinte ou de l'absence

43 Le Conseil constitutionnel semblant examiner d'office dans cette situation le respect des règles ou principes inhérents à l'identité constitutionnelle de la France. Ainsi, dans la décision du 17 décembre 2010, M. Kamel D. Transposition d'une directive] ( ${ }^{\circ}$ 2010-79 QPC, Rec., p. 406), il indique «qu'en l'absence de mise en cause d'une règle ou d'un principe inhérent à l'identité constitutionnelle de la France, [il] n'est pas compétent pour contrôler la conformité aux droits et libertés que la Constitution garantit de dispositions législatives qui se bornent à tirer les conséquences nécessaires de dispositions inconditionnelles et précises d'une directive de l'Union européenne » (cons. 3). Il ajoute qu'en l'espèce les dispositions législatives contestée «ne mettent en cause aucune règle ni aucun principe inhérent à l'identité constitutionnelle de la France » (cons. 4). 
d'atteinte à l'identité nationale, en fonction de l'interprétation retenue de la disposition législative nécessaire au droit de l'Union. Sa fonction conciliatrice sera ici significative. Le Conseil constitutionnel pourrait peut-être préférer à cette première voie un autre renvoi préjudiciel, en appréciation de validité, et renvoyer ainsi à la Cour de justice le soin de résoudre le conflit d'une autre manière.

\section{B - L’usage neutralisant du renvoi préjudiciel en appréciation de validité}

Le renvoi préjudiciel en appréciation de validité peut conduire à faire directement résoudre par la Cour de justice de l'Union européenne un conflit entre un acte de droit dérivé et la Constitution. Le conflit nait au niveau national et sera résolu par les juges de Luxembourg au niveau de l'Union européenne. Plus précisément, la Cour peut être conduite à résoudre soit un conflit entre le droit dérivé et des normes constitutionnelles ordinaires, soit un conflit de ce même droit dérivé avec l'identité nationale de la France. Le premier cas est envisageable dès lors qu'il existe au niveau du droit primaire de l'Union des règles équivalentes à celles existantes dans la Constitution; le second, car l'article $4 \int 2$ du TUE précise que l'Union européenne respecte l'identité nationale des Etats et fait ainsi entrer dans le domaine du droit de l'Union cette notion d'identité nationale. Dans les deux cas, le jugement de constitutionnalité ne revient plus à la juridiction nationale, qui n'est plus alors qu'un lanceur d'alerte, mais au juge de l'Union, ce qui garantit le respect du droit de l'Union. Les modalités de mise en œuvre de ces deux cas sont différentes. Le contrôle de constitutionnalité est externalisé devant le juge de Luxembourg.

La première situation est la plus complexe. En principe en effet, le Conseil constitutionnel est incompétent pour contrôler une loi nécessaire au droit de l'Union au regard des normes constitutionnelles ordinaires. Il ne peut le faire qu'au regard des règles ou principes inhérents à l'identité constitutionnelle de la France. Dans la décision du 17 décembre 2010, M. Kamel D., à propos d'une loi de transposition d'une directive, il a ainsi jugé « qu'en l'absence de mise en cause d'une règle ou d'un principe inhérent à l'identité constitutionnelle de la France, le Conseil constitutionnel n'est pas compétent pour contrôler la conformité aux droits et libertés que la Constitution garantit de dispositions législatives qui se bornent à tirer les conséquences nécessaires de dispositions inconditionnelles et précises d'une directive de l'Union européenne ${ }^{44}$. Autrement dit, la situation dans laquelle le Conseil constitutionnel aurait à juger de la conformité d'une loi nécessaire au droit de l'Union à une norme constitutionnelle ordinaire ne peut pas se produire, du moins si la jurisprudence du Conseil constitutionnel est respectée. Pour être plus précis, il faut sans doute distinguer entre le contrôle a priori et la QPC.

Dans le cadre du contrôle a priori, il est possible d'envisager une erreur des requérants ou un manque d'attention les conduisant à contester une disposition de loi nécessaire à la transposition

44 C.C., n 2010-79 QPC, 17 décembre 2010, M. Kamel D. [Transposition d'une directive], cons. 3. 
de dispositions inconditionnelles et précises d'une directive au regard de normes constitutionnelles ordinaires, sans que ne soit mentionnée l'identité nationale. Dans une telle situation, le Conseil constitutionnel, s'il n'est pas compétent pour exercer un contrôle de constitutionnalité $^{45}$, pourrait cependant soulever une question préjudicielle en appréciation de validité devant la Cour de justice si le droit de l'Union contient une règle ou un principe substantiellement équivalent à la norme constitutionnelle ordinaire invoquée. A l'évidence, il ne saurait s'agir que de normes constitutionnelles de droits fondamentaux. L'immunité constitutionnelle contentieuse du droit de l'Union serait ainsi compensée par un contrôle de la Cour de justice, la censure éventuelle de cette dernière pouvant justifier ensuite, devant le Conseil constitutionnel, une censure de la loi.

Dans le cadre de la QPC, la situation change dans la mesure où le Conseil constitutionnel a moins de chance encore d'être saisi de la question du respect par des lois nécessaires au droit de l'Union de normes constitutionnelles ordinaires en raison du filtre des juridictions de droit commun. Les juridictions de transmission et de renvoi, à moins qu'elles ne maîtrisent pas la jurisprudence constitutionnelle ou qu'elles omettent l'origine européenne de la disposition contestée ${ }^{46}$, ne sauraient saisir le Conseil constitutionnel d’une telle question qui ne relève pas de sa compétence.

De plus, la Cour de justice a spécifiquement envisagé cette situation dans l'arrêt du Melki et Abdeli, en imposant aux juges du filtre de la saisir d'une question préjudicielle en appréciation de validité. Elle a jugé alors qu'« avant que le contrôle incident de constitutionnalité d'une loi dont le contenu se limite à transposer les dispositions impératives d'une directive de l’Union puisse s'effectuer par rapport aux mêmes motifs mettant en cause la validité de la directive, les juridictions nationales, dont les décisions ne sont pas susceptibles d'un recours juridictionnel de droit interne, sont, en principe, tenues, en vertu de l'article 267, troisième alinéa, TFUE, d'interroger la Cour de justice sur la validité de cette directive et, par la suite, de tirer les conséquences qui découlent de l'arrêt rendu par la Cour à titre préjudiciel, à moins que la juridiction déclenchant le contrôle incident de constitutionnalité n'ait elle-même saisi la Cour de justice de cette question sur la base du deuxième alinéa dudit article ${ }^{47}$. La situation visée est bien celle de la QPC et de l'invocation de la violation d'une disposition constitutionnelle qui trouve un équivalent dans le droit de l'Union par une disposition nationale transposant les dispositions impératives d'une directive. La saisine de la Cour de justice et sa décision sur le renvoi doivent

45 Tout en n'exerçant pas de contrôle de constitutionnalité au regard des normes constitutionnelles ordinaires, il est possible de penser que le Conseil constitutionnel pourrait exercer d'office un contrôle du respect des règles et principes inhérents à l'identité constitutionnelle de la France, comme ce fût le cas dans la décision du 17 décembre 2010, M. Kamel D. (précit.). Sans anticiper sur ce que le Conseil constitutionnel peut faire dans cette dernière situation (voir infra), le choix de soulever une question préjudicielle en appréciation de validité au regard des normes constitutionnelles ordinaires, trouvant un équivalent dans le droit primaire de l'Union européenne, ou des règles ou principes inhérents à l'identité constitutionnelle de la France dépendra de ce que le droit dérivé de l'Union contesté met potentiellement en cause l'une ou l'autre de ces deux catégories de normes constitutionnelles.

46 Ce qui était en l'occurrence le cas dans la décision de renvoi du Conseil d'Etat dans l'affaire Kamel D. : C.E., 8 octobre $2010, n^{\circ} 338505$.

47 C.J., 22 juin 2010, Aquiz. Melki et Sélim Abdeli, C-188/10 et c-189/10, précit., \56. 
avoir lieu avant le contrôle de constitutionnalité. Les commentaires sous la décision du Conseil constitutionnel Kamel D. avaient envisagé cette situation et, tout en reconnaissant que le renvoi et le jugement de la Cour devaient précéder le jugement du Conseil constitutionnel, soutenaient que les juridictions de renvoi pouvaient simultanément soulever les deux questions, question préjudicielle en appréciation de validité et QPC, à charge pour le Conseil constitutionnel d'attendre le jugement de la Cour de justice avant de juger de la constitutionnalitée ${ }^{4}$. Il convient cependant de remarquer que s'il existe une équivalence dans le droit de l'Union du principe constitutionnel invoqué contre le droit national nécessaire au droit de l'Union, il ne peut s'agir que d'une norme constitutionnelle ordinaire qui ne relève donc pas de l'identité constitutionnelle, situation contentieuse que le Conseil constitutionnel ne saurait pas connaitre.

Lorsqu'est soulevé dans le cadre de la QPC le moyen tiré de la violation par une disposition nationale dont le contenu est imposé par le droit de l'Union d'une disposition constitutionnelle ordinaire, c'est-à-dire en dehors de l'identité nationale, qui trouve son équivalent dans le droit de l'Union, si le droit national interdit en principe un renvoi ou une transmission de la QPC, le Conseil constitutionnel étant incompétent pour la juger, le droit de l'Union, selon l'interprétation de la Cour (même si elle est contenue seulement dans les motifs...), impose un renvoi préjudiciel en appréciation de validité à la Cour de justice. Le Conseil constitutionnel ne saurait donc en principe connaittre de telles questions, le renvoi à la Cour de justice se faisant avant qu'il ne soit saisi. S’il était néanmoins saisi, il pourrait lui-même, tout en affirmant son incompétence, renvoyer à la Cour de justice le soin de trancher la question. L'immunité contentieuse nationale est compensée par la saisine de la Cour de justice, le droit de l'Union suppléant ainsi cette immunité dont il est, en l'occurrence, à l'origine. C'est bien le respect du droit de l'Union et la couverture constitutionnelle générale offerte à celui-ci par l'article 88-1 de la Constitution qui interdit au juge constitutionnel de remettre en cause le droit dérivé de l'Union en vigueur au regard des normes constitutionnelles ordinaires.

La seconde situation est plus simple même si sa portée symbolique peut heurter la sensibilité des âmes souverainistes, si l'on suppose, bien sûr, que les souverainistes ont une âme. Elle correspond à ce qui doit se produire si la jurisprudence du Conseil constitutionnel est respectée, à savoir si l'identité constitutionnelle est invoquée contre une disposition législative nécessaire au droit de l'Union. Dans cette hypothèse, le renvoi préjudiciel en appréciation de validité est possible précisément pour demander à la Cour d'invalider l'acte de l'Union éventuellement contraire à cette identitée ${ }^{49}$. Dans la mesure où l'Union respecte l'identité nationale des Etats, la Cour de justice pourrait être en mesure d'invalider un acte de droit de l'Union dérivé contraire à l'identité nationale d'un Etat. Le juge européen ne s'est pas encore prononcé en ce sens mais l'on peut considérer qu'une atteinte par le droit dérivé de l'Union à l'identité nationale des Etats est

48 Commentaires en ligne sous C.C., n² 2010-79 QPC, 17 décembre 2010, M. Kamel D. [Transposition d'une directive], p. 8.

49 Le juge constitutionnel peut d'ailleurs, dans cette même situation, aussi procéder à un renvoi préjudiciel en interprétation, voir supra, \II, A. 
une méconnaissance de l'article 4 \ 2 du TUE susceptible d'être sanctionnée par la Cour de justice. Celle-ci vérifie déjà que les Etats invoquent à juste titre le respect de leur identité nationale pour échapper à l'application du droit de l'Union dans le cadre du renvoi préjudiciel en interprétation ${ }^{50}$. Rien n'empêche de penser, sous réserve des choix de politique jurisprudentielle, que l'identité nationale puisse également rentrer dans les exigences de la légalité du droit de l'Union. Si le juge constitutionnel national procédait à un renvoi en ce sens, il ne serait plus le juge du respect de l'identité nationale, tout en étant l'instrument permettant de la faire respecter par le renvoi à la Cour de justice. Le juge national renvoie ainsi à la Cour de justice le soin d'apprécier la régularité du droit de l'Union dérivé à l'identité nationale dans le respect du droit de l'Union. Cette lecture peut apparaitre iconoclaste, elle n'en semble pas moins possible. Toute la difficulté d'ordre politique qu'elle recèle consiste à déposséder le juge constitutionnel de la compétence pour exercer un contrôle du respect de l'identité nationale et à admettre une lecture de l'identité nationale en dehors de l'Etat.

La portée du renoncement à une lecture nationale de l'identité de la France ne doit pas pour autant être surestimée. Si le Conseil constitutionnel oppose seul l'identité nationale de la France à un acte interne nécessaire à l'application du droit de l'Union, sa décision pourra toujours être remise en cause par la Cour de justice par la voie d'un recours en manquement. Ce sera alors cette dernière qui sera en mesure d'avoir le dernier mot sur l'interprétation de la conformité à l'identité nationale de la France. Face à une supposée méconnaissance de l'identité nationale soulevée devant le juge constitutionnel, tout est question de choix. Il peut s'en remettre, de manière neutre vis-à-vis du droit de l'Union, à l'appréciation de la Cour de justice par un renvoi préjudiciel en appréciation de validité, ou juger seul en espérant que la Cour de justice ne soit pas ultérieurement saisie de la même question par la voie d'un recours en manquement, qu'elle le déjuge et que la France soit alors condamnée pour violation du droit de l'Union. Si le juge constitutionnel s'en remet à l'appréciation de la Cour par la voie préjudicielle, la portée potentiellement conflictuelle de la jurisprudence Confiance dans l'économie numérique est annihilée. Il est encore nécessaire de s'interroger sur le juge susceptible de procéder à un tel renvoi dans le cadre de la QPC. Le Conseil constitutionnel peut renvoyer en dernier lieu mais les juges de renvoi ou de transmission peuvent également le faire avant lui. Il existe ainsi une concurrence des juges susceptibles de procéder au renvoi préjudiciel. L'intervention du Conseil constitutionnel n'interviendrait alors qu'en dernier recours, le conflit pouvant être résolu avant son intervention. En pratique, les juridictions de droit commun pourraient d'ailleurs priver le Conseil constitutionnel de sa compétence de renvoi en usant elle-même du renvoi préjudiciel, dans le même sens que le Conseil constitutionnel l'a fait dans le cadre de l'affaire Jeremy $P^{51}$ ou dans les autres situations qui ont été ici envisagées. L’usage du renvoi préjudiciel par le Conseil

50 Voir par exemple: C.J., 16 avril 2013, Anton Las c. PSA Antwerp NV, aff. C-202/11; 22 décembre 2010 , SaynWittgenstein c. Landeshauptmann von Wien, aff. C-208/09, Rec. 2010, p. I-13693.

51 Le principe et/ou les modalités du contrôle éventuel du Conseil constitutionnel seraient toujours déterminés par la Cour de justice en fonction du lien existant entre le droit national et le droit de l'Union, mais sur renvoi des juges de transmission ou de renvoi et non pas du Conseil constitutionnel. 
constitutionnel dans le cadre de la QPC est de la sorte conditionné par celui qu'en auront les juges de droit commun avant ou au moment de transmettre ou de renvoyer la question au juge constitutionnel.

Au-delà du principe même de la reconnaissance de la possibilité pour le Conseil constitutionnel de renvoyer une question préjudicielle à la Cour de justice, qui pourrait apparaitre comme anecdotique, ce sont les potentialités d'utilisation de ce renvoi qui sont susceptibles d'affecter en profondeur le contentieux constitutionnel (français) du droit de l'Union européenne. L'ultime limite, du moins visible, au respect du droit de l'Union tirée du respect de l'identité nationale pourrait être envisagée de manière pacifique entre le Conseil constitutionnel et la Cour de justice dans le respect du droit constitutionnel et du droit de l'Union. Qui opposera encore l'existence de contre-limites?

\author{
Xavier MAGNON \\ Professeur à l'Université de Toulouse 1 Capitole \\ Institut Maurice Hauriou
}

